\title{
Diagnostic Value of the Second Lumbrical- Interosseous Distal Motor Latency Comparison Test in Severe Carpal Tunnel Syndrome
}

\author{
SangHun Lee, MD, DongHyun Kim, MD, Hee-Mun Cho, MD, \\ Ho-Sung Nam, MD, Dong-Sik Park, MD
}

Department of Rehabilitation Medicine, Hallym University Kangdong Sacred Heart Hospital, Hallym University College of Medicine, Seoul, Korea

Objective To examine the usefulness of the second lumbrical-interosseous (2L-INT) distal motor latency (DML) comparison test in localizing median neuropathy to the wrist in patients with absent median sensory and motor response in routine nerve conduction studies.

Methods Electrodiagnostic results from 1,705 hands of patients with carpal tunnel syndrome (CTS) symptoms were reviewed retrospectively. All subjects were evaluated using routine nerve conduction studies: median sensory conduction recorded from digits 1 to 4, motor conduction from the abductor pollicis brevis muscle, and the 2L-INT DML comparison test.

Results Four hundred and one hands from a total of 1,705 were classified as having severe CTS. Among the severe CTS group, 56 hands (14.0\%) showed absent median sensory and motor response in a routine nerve conduction study, and, of those hands, $42(75.0 \%)$ showed an abnormal 2L-INT response.

Conclusion The 2L-INT DML comparison test proved to be a valuable electrodiagnostic technique in localizing median mononeuropathy at the wrist, even in the most severe CTS patients.

Keywords Carpal tunnel syndrome, Nerve conduction, Second lumbrical-interosseous distal motor latency comparison test

Received May 15, 2015; Accepted July 22, 2015

Corresponding author: Dong-Sik Park

Department of Rehabilitation Medicine, Hallym University Kangdong Sacred Heart Hospital, Hallym University College of Medicine, 150 Seongan-ro, Gangdong-gu, Seoul 05355, Korea

Tel: +82-2-2224-2660, Fax: +82-2-2225-2789, E-mail: don@hallym.or.kr

(a) This is an open-access article distributed under the terms of the Creative Commons Attribution Non-Commercial License (http://creativecommons. org/licenses/by-nc/4.0) which permits unrestricted noncommercial use, distribution, and reproduction in any medium, provided the original work is properly cited.

Copyright $\odot 2016$ by Korean Academy of Rehabilitation Medicine

\section{INTRODUCTION}

Carpal tunnel syndrome (CTS) is the most common peripheral neuropathy, and is caused by an entrapment of the median nerve at the wrist. Electrophysiologic study is commonly used in confirming the diagnosis of CTS, with several parameters and techniques being used [19]. General measurements are made by sensory nerve conduction at the index and middle finger, and by motor conduction from the abductor pollicis brevis (APB) 
muscle [6]. Additional studies have been carried out such as measurement of onset latency difference at the wrist and palm; comparison of latency differences between the median-radial and median-ulnar sensory nerves from thumb and ring finger; and comparison of onset latencies of compound muscle action potentials (CMAP) of second lumbrical (2L) muscles and interosseous (INT) muscles in the palm. They have been used to increase the sensitivity in diagnosis of CTS [1-8].

Several reports have described the diagnostic value of a 2L-INT test in CTS [1-8]. In 1992, Preston and Logigian [2] reported the 2L-INT distal motor latency (DML) difference to be a sensitive measurement for confirmation of CTS diagnosis. The advantage of the technique is described as follows: both muscles can be easily recorded from the same active electrode; nerve endings innervating both muscles are similar in diameter; the surrounding temperature is equivalent; the identical distance to each muscle allows direct comparison of distal motor latencies; and the $2 \mathrm{~L}$ muscles are relatively spared in CTS [2].

Boonyapisit et al. [4], Brannegan and Bartt [5] and Inukai et al. [6] reported that 2L-CMAP is maintained even in the most severe cases of CTS when response is absent in a motor conduction study of the APB muscle. Meena et al. [7] reported that the sensitivity of 2L-INT DML difference was $90 \%$ in a severe CTS group, and was diagnostic in 7 out of 8 cases where both palm-wrist distal sensory latency difference and median DML were not obtained due to the loss of sensory and motor response.

This study aimed to investigate the diagnostic efficacy of the 2L-INT DML comparison test in localizing median mononeuropathy at the wrist in patients who present absent median sensory response, recorded from digits 1 to 4 , and median motor response from the APB muscle in routine nerve conduction studies.

\section{MATERIALS AND METHODS}

\section{Subjects}

Between March 2005 and March 2014, we studied 1,705 hands of patients with clinical symptoms and signs suggestive of CTS who were referred to Kangdong Sacred Heart Hospital for electrophysiologic study. Retrospective chart reviews were carried out, including those of electrophysiologic test results. All electrophysiologic results were classified into mild, moderate, and severe groups according to Padua's neurophysiologic staging of CTS [10]. We further categorized the severe CTS patients with absent median sensory and motor responses as determined by routine conduction studies into an extremely severe hand group. Patients with co-morbid cervical radiculopathy, polyneuropathy and/or any other peripheral neuropathy were excluded.

\section{Methods}

The electrophysiologic study was performed using either Viking IV (Nicolet, Madison, WI, USA) or Cadwell Sierra Wave (Cadwell Laboratories, Kennewick, WA, USA). Disposable silver chloride discs of $2 \mathrm{~cm}$ in diameter were used. Palmar skin temperature was set above $32^{\circ} \mathrm{C}$ according to the guideline recommended by Dumitru and Zwarts [9] and the temperature used in previous studies $[4,6,10]$. For the motor conduction study, the sweep speed, gain and filter were set to $5 \mathrm{~ms} /$ division, 1-5 mV/ division and $10 \mathrm{~Hz}-10 \mathrm{kHz}$, respectively. Identical settings were always used to compare the 2L-INT latencies within the subject.

2L-INT CMAPs were recorded using the same technique used by Preston and Logigian [2]: the active electrode was placed slightly lateral to the midpoint of the third metacarpal bone and the recording electrode was placed distally over the bony prominence of the proximal interphalangeal joint of the second digit. The nerves were stimulated at the wrist, each with identical distance from the active electrode. The median nerve was stimulated between the palmaris longus tendon and the flexor carpi radialis tendon, and the ulnar nerve was stimulated just lateral to the flexor carpi ulnaris tendon (Fig. 1A, B). With a gradual increase of intensity to avoid co-stimulation of both nerves at the wrist, supra-maximal 2L-INT CMAPs were recorded, and each was identified by their characteristic waveforms; the 2L CMAP waveform usually had a fluent curve with lower amplitude and longer duration than the INT CMAP [4] (Fig. 2).

According to the previous study reported by Preston and Logigian [2], the 2L-INT latency difference in normal subjects was equal to or less than $0.40 \mathrm{~ms}$ when stimulated from the same distance sites. Therefore, the abnormal 2L-INT latency difference was defined at $>0.40 \mathrm{~ms}$. The prevalence of abnormal 2L-INT DML differences among the group was analyzed. 

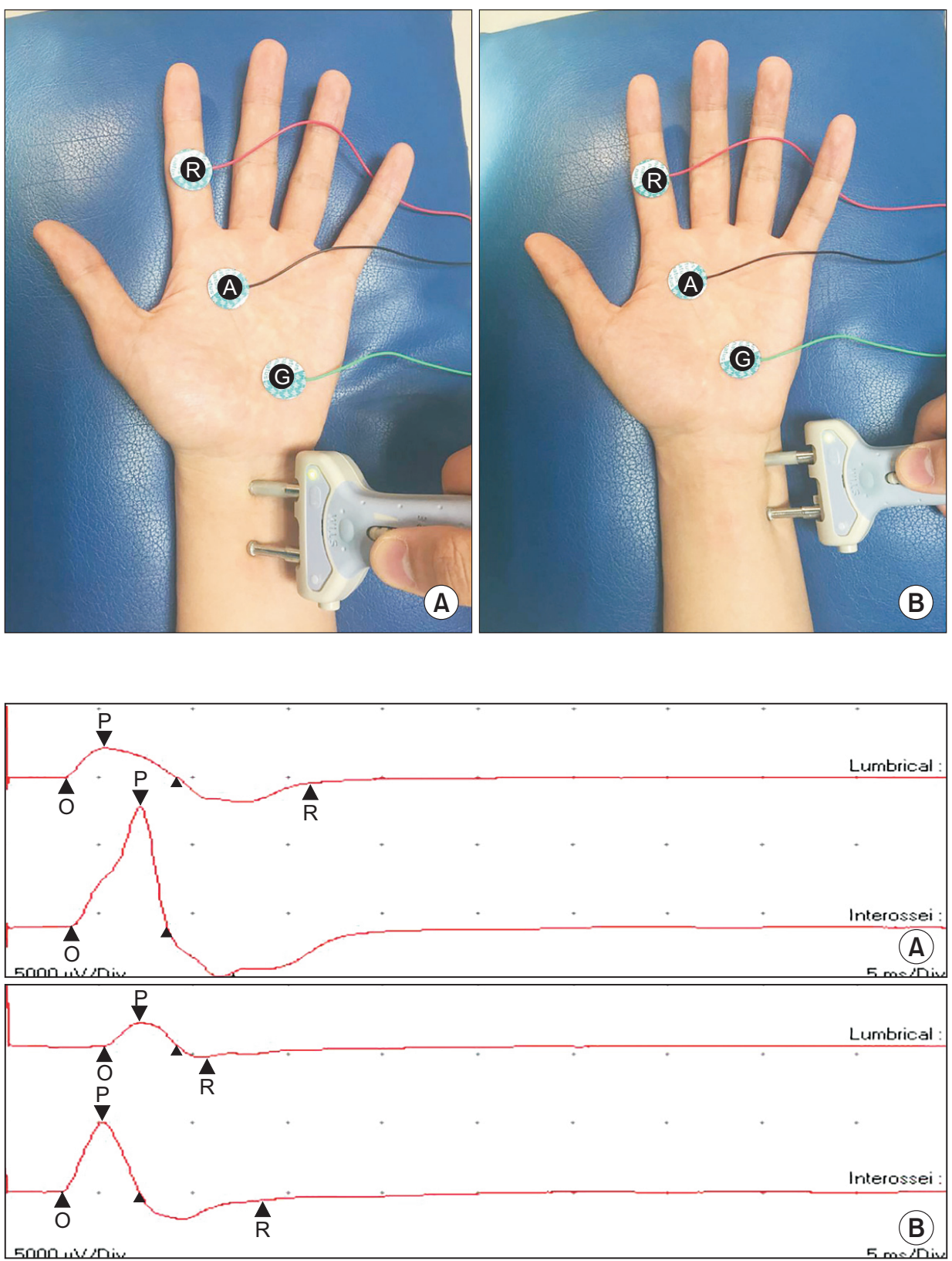

Fig. 1. Recording and stimulation site of $2 \mathrm{~L}$ (A) and INT (B). 'A' is active electrode, ' $R$ ' reference electrode, and ' $\mathrm{G}$ ' ground electrode. $2 \mathrm{~L}$, second lumbrical; INT, interosseous.

Fig. 2. The second lumbrical-interosseous distal motor latency comparison test showing (A) normal latency difference $(0.2 \mathrm{~ms})$ and (B) prolonged latency difference (2.1 ms).

\section{RESULTS}

Among the 401 hands that were subsequently proven to have severe CTS according to Padua's neurophysiologic staging, 56 hands in 45 patients fell into the extremely severe CTS group (Fig. 3). The incidence of severe CTS with absent sensory and motor studies was $14 \%$. The mean age of the group was $52.76 \pm 9.99$ years (range, $32-84$ years), and the female/male ratio was 10:1 (41 female and 4 male).

Forty-two hands out of 56 hands (75.0\%) showed an ab- normal 2L-INT DML difference with significant prolongation of 2L DML. Fourteen hands revealed absent median motor response from $2 \mathrm{~L}$, as did the median motor recording from the APB muscle. The average and range of each parameter obtained from the electrophysiologic results of 42 hands are listed in Table 1. Mean 2L DML was $7.7 \pm 2.77 \mathrm{~ms}$ (range, 3.8-16 ms) and mean 2L CMAP amplitude was $0.73 \pm 0.56 \mathrm{mV}$ (range, $0.11-2.9 \mathrm{mV}$ ); mean INT DML was $3.1 \pm 0.36 \mathrm{~ms}$ (range, $2.3-3.8 \mathrm{~ms}$ ) and mean INT CMAP amplitude was $4.64 \pm 1.48 \mathrm{mV}$ (range, 2.8-9.14 $\mathrm{mV}$ ); mean 2L-INT DML difference was $4.53 \pm 2.63 \mathrm{~ms}$ 


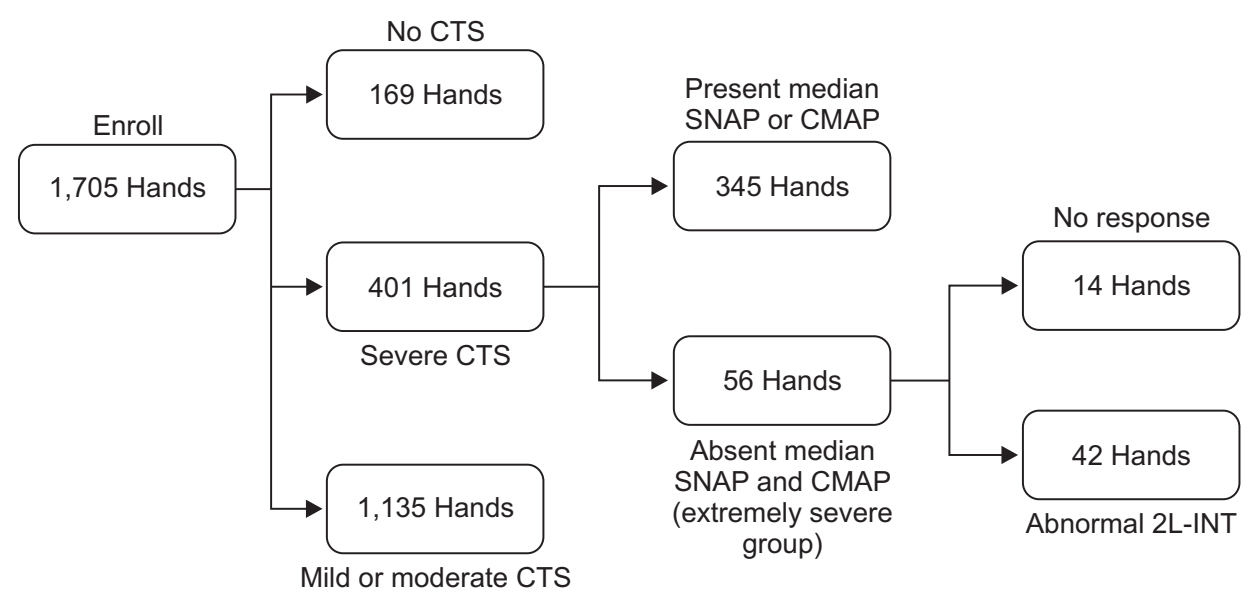

Fig. 3. Flowchart defining the extremely severe group. The whole experimental group was first classified according to Padua's neurophysiologic staging of CTS. Among the severe CTS group (401 hands), the extremely severe group was sorted by those whose median SNAP and CMAP were absent (56 hands). Forty-two hands in the extremely severe group showed abnormal 2L-INT DML comparison and 14 hands showed no response in 2L. CTS, carpal tunnel syndrome; SNAP, sensory nerve action potential; CMAP, compound muscle action potential; 2L, second lumbrical; INT, interosseous; DML, distal motor latency.

Table 1. Summary of 2L-INT study in the extremely severe CTS group

\begin{tabular}{lc}
\hline \multicolumn{1}{c}{ Parameter } & Value \\
\hline 2L-INT DIFF (ms) & $4.53 \pm 2.63(0.5-10.2)$ \\
2L DML (ms) & $7.7 \pm 2.77(3.8-16)$ \\
INT DML (ms) & $3.1 \pm 0.36(2.3-3.8)$ \\
2L AMP (mV) & $0.73 \pm 0.56(0.11-2.9)$ \\
\hline INT AMP (mV) & $4.64 \pm 1.48(2.8-9.14)$ \\
\hline
\end{tabular}

Values are presented as mean \pm standard deviation (range). The compound muscle action potentials from the $2 \mathrm{~L}$ muscle could not be obtained in 16 cases; thus, $n=42$ for 2L-INT DIFF, 2L DML, and 2L AMP; $\mathrm{n}=56$ for INT DML and INT AMP.

CTS, carpal tunnel syndrome; 2L-INT, second lumbrical and interosseous; DIFF, difference; DML, distal motor latency; AMP, amplitude.

(range, 0.5-10.2 ms).

The sensitivities of the 2L-INT DML comparison test were $88.8 \%$ and $75.0 \%$ in the whole experimental group and the extremely severe group, respectively, and were calculated as followed: among the whole experimental group, 1,546 hands were diagnosed as having CTS from electrophysiologic study and 1,363 hands were shown to be abnormal according to the 2L-INT DML comparison test; among 56 hands of the extremely severe group, 42 hands were revealed to be abnormal according to the
2L-INT DML comparison test. The specificity of the test (96.4\%) could only be obtained from the population and not from the extremely severe group due to lack of disease-free hands; among the 169 hands with negative CTS findings according to electrophysiologic study, 165 hands showed normal findings in the 2L-INT DML comparison test.

Needle electromyography revealed abnormal spontaneous activity in the APB muscle in all studied hands and other proximal median-innervated muscles (flexor pollicis longus, flexor digitorum superficialis, flexor carpi radialis, and pronator teres) were normal.

\section{DISCUSSION}

CTS is the most common entrapment neuropathy of the upper limb, and electrophysiologic study still remains as the gold standard for its diagnosis. In addition to routine the median sensory response recorded from digits 1 to 4 and median motor response from the APB muscle, further comparison conduction studies such as medianradial sensory latency difference to the thumb, medianulnar sensory latency difference to the ring finger, median-ulnar mixed nerve latency difference to the palm, and 2L-INT DML comparison are being used to aid in the confirmative diagnosis of CTS [1-8].

Chronic compression of the median nerve at or around 
the wrist induces Wallerian degeneration and axonal injury, leading to atrophy of the thenar muscles and absent response in routine electrophysiologic study in the advanced stage $[3,9]$. In such cases, even if CTS is strongly suspected, it is very challenging to confirm the diagnosis of the median neuropathy at the wrist. Due to absent median sensory responses from digits 1 to 4 , out of the comparison tests listed above, only the 2L-INT DML comparison test is applicable. Therefore, a 2L-INT DML comparison can be useful in localizing median mononeuropathy at the wrist in extremely severe groups.

Previous studies already revealed the usefulness of $2 \mathrm{~L}$ recording and 2L-INT DML comparisons in severe CTS patients with non-responding median sensory and motor results. Reported sensitivities of the 2L-INT DML comparison test and 2L recording in extremely severe groups were as follows: $86.1 \%$ by Loscher et al. [11], $92.8 \%$ by Boonyapist et al. [4], and $77 \%$ by Brannegan et al. [5]. According to a study by Kaul and Pagel [12], the 2L-INT comparison had higher obtainability as compared to the routine median thenar in patients with absent median sensory response ( $100 \%$ versus $95 \%$ ). In our study, the mean 2L-INT DML difference was $4.53 \pm 2.63$, which was evidently delayed. In addition, it enabled the confirmation of median mononeuropathy at the wrist in $75 \%$ of patients with absent median sensory and motor response in routine nerve conduction studies.

In 1981, Yates et al. established that at a late stage in the entrapment of the median nerve, the motor fibers to the lumbrical muscles may be relatively preserved compared to motor fibers to the thenar muscles [13]. They suggested that the motor fibers to the lumbrical muscles present better tolerance to compression against the flexor retinaculum, as they lie deeper in the carpal tunnel compared to thenar motor fibers. This is supported by intraneural investigations conducted by Sunderland, who revealed that the funiculus carrying the $2 \mathrm{~L}$ fibers is more centrally located in relation to thenar fibers and sensory fibers [14]. Planitzer et al. reported that the median nerve motor fascicle to the $2 \mathrm{~L}$ was mostly located on the ulnar dorsal side [15]. Thus, the fibers located around the periphery of the nerve, near the flexor retinaculum, (motor fibers to thenar muscles and sensory fibers) are more susceptible to compression and axonal damage than more dorsally and centrally located lumbrical fibers, advocating the diagnostic value of the 2L-INT DML com- parison test in extremely severe CTS.

The prevalence of extremely severe hand groups among confirmed CTS cases could be found in the following studies: $2.4 \%$ of 975 cases in a study by Boonyapisit et al. [4]; 11.5\% of 312 cases in a study by Loscher et al. [11]; $2 \%$ of 1,123 hands in a study by Padua et al. [16]; and $3 \%$ in 66 cases in a study by Sheean et al [17]. This study included the largest CTS cases and the highest number of severe CTS hands with absent median sensory and motor responses, and found 56 hands among 1,536 confirmed CTS cases, a prevalence of $3.6 \%$.

Limitations of this study include an unequal male to female ratio and the relatively small sample size. Although the number of studied hands was the largest as compared to the previous results, further study with a larger sample size and equal sex ratio needs to be conducted to standardize the application of the 2L-INT DML comparison test in the general population.

In conclusion, this study was performed to illustrate the 2L-INT DML comparison test as an invaluable electrodiagnostic technique in improving localization and diagnostic certainty of median neuropathy at the wrist, even for the most severe CTS cases. It is easy to perform and shows high sensitivity even when routine conduction studies fail to produce meaningful results.

\section{CONFLICT OF INTEREST}

No potential conflict of interest relevant to this article was reported.

\section{REFERENCES}

1. Lee HJ, Kwon HK, Kim DH, Pyun SB. Nerve conduction studies of median motor nerve and median sensory branches according to the severity of carpal tunnel syndrome. Ann Rehabil Med 2013;37:254-62.

2. Preston DC, Logigian EL. Lumbrical and interossei recording in carpal tunnel syndrome. Muscle Nerve 1992;15:1253-7.

3. Park DS, Nam HS, Kim DH, Choi EH, Jun AY, Lee SE. Diagnostic usefulness of the second lumbrical and interosseous recording in severe carpal tunnel syndrome. J Korean EMG Electrodiagn Med 2009;11:73-7.

4. Boonyapisit K, Katirji B, Shapiro BE, Preston DC. Lumbrical and interossei recording in severe carpal 
tunnel syndrome. Muscle Nerve 2002;25:102-5.

5. Brannegan R, Bartt R. Second lumbrical muscle recordings improve localization in severe carpal tunnel syndrome. Arch Phys Med Rehabil 2007;88:259-61.

6. Inukai T, Uchida K, Kubota C, Takamura T, Nakajima $\mathrm{H}, \mathrm{Baba} \mathrm{H}$. Additional method for diagnosis of carpal tunnel syndrome: value of the second lumbricalinterossei test (2L-INT). Hand Surg 2013;18:49-52.

7. Meena AK, Srinivasa Rao B, Sailaja S, Mallikarjuna M, Borgohain R. Second lumbrical and interossei latency difference in carpal tunnel syndrome. Clin Neurophysiol 2008;119:2789-94.

8. Ahn MK, Kim SJ. Diagnostic value of median-ulnar motor difference recorded on lumbrical and interosseous in carpal tunnel syndrome. J Korean Acad Rehabil Med 1994;18:35-44.

9. Dumitru D, Zwarts MJ. Nerve conduction studies. In: Dumitru D, Amato AA, Zwarts M, editors. Electrodiagnostic medicine. 2nd ed. Philadelphia: Hanley \& Belfus; 2002. p. 159-223.

10. Padua L, Lo Monaco M, Padua R, Gregori B, Tonali P. Neurophysiological classification of carpal tunnel syndrome: assessment of 600 symptomatic hands. Ital J Neurol Sci 1997;18:145-50.

11. Loscher WN, Auer-Grumbach M, Trinka E, Ladurner
G, Hartung HP. Comparison of second lumbrical and interosseous latencies with standard measures of median nerve function across the carpal tunnel: a prospective study of 450 hands. J Neurol 2000;247:530-4.

12. Kaul MP, Pagel KJ. Median sensory nonresponders in carpal tunnel syndrome workup. Arch Phys Med Rehabil 2002;83:1120-2.

13. Yates SK, Yaworski R, Brown WF. Relative preservation of lumbrical versus thenar motor fibres in neurogenic disorders. J Neurol Neurosurg Psychiatry 1981;44:76874.

14. Sunderland S. The intraneural topography of the radial, median and ulnar nerves. Brain 1945;68:243-99.

15. Planitzer U, Steinke H, Meixensberger J, Bechmann I, Hammer N, Winkler D. Median nerve fascicular anatomy as a basis for distal neural prostheses. Ann Anat 2014;196:144-9.

16. Padua L, Padua R, Lo Monaco M, Aprile I, Tonali P. Multiperspective assessment of carpal tunnel syndrome: a multicenter study. Italian CTS Study Group. Neurology 1999;53:1654-9.

17. Sheean GL, Houser MK, Murray NM. Lumbricalinterosseous latency comparison in the diagnosis of carpal tunnel syndrome. Electroencephalogr Clin Neurophysiol 1995;97:285-9. 